\title{
Muscular Adaptation to Exercise in Sport Horses
}

\author{
Felipe Gomes Ferreira Padilha* and Ana Maria Reis Ferreiraa \\ Graduate Program in Veterinary Medicine (Clinic and Animal Reproduction), Vital Brazil
}

*Corresponding author: Felipe Gomes Ferreira Padilhaa, Graduate Program in Veterinary Medicine (Clinic and Animal

Reproduction), Vital Brazil

\begin{tabular}{|c|c|}
\hline ARTICLE INFO & ABSTRACT \\
\hline Received: 彗 May 07, 2019 & Citation: Felipe Gomes Ferreira P, Ana Maria Reis F. Muscular Adaptation to Exercise in \\
\hline Published: 㸷 May 14, 2019 & Sport Horses. Biomed J Sci \& Tech Res 18(1)-2019. BJSTR. MS.ID.003087. \\
\hline
\end{tabular}

\section{Mini Review}

Studies about muscular adaptation to training date from the beginning of percutaneous muscle biopsies in horses. Guy \& Snow (1977) evaluated samples collected after an exercise program in horses and, later, a period without training. The adaptation of equine locomotor muscles to a training protocol depends on the characteristic of the exercise, fiber type, oxidative potential and structural rearrangements of muscle fibers. The sectional area of the muscle fiber types depends on the age and gender of the equines and the intensity and duration of training [1]. Resistance equine training induces a reversible transition in the composition of the myosin heavy chain in the following order: IIX $\diamond$ IIA $\diamond \mathrm{I}$, which is accompanied by changes in muscle metabolic capacity [2]. Increased muscle fiber area, number of capillaries and mitochondrial volume indicating a progressive increase in aerobic capacity with increasing workload after a training program are also important findings [3]. During the evaluation of different types of conditioning programs in muscle variables in sport horses, adaptive responses consisted of a significant increased number of mitochondria and myofibrils, as well as myofibrillar ATPase type IIA hypertrophy and the reduction in the number of fibers of the type IIX, low oxidative. Together, these changes are compatible with increased oxidative capacity and muscle strength [4]. Low- or moderate-intensity and long-duration exercises are more effective in improving the muscular resources associated with vigor and strength in athletic horses than in high intensity and short duration exercises [5].

Both intensity and duration of exercise are critical factors in determining the nature and magnitude of short-term training adaptations. Conversions of myosin heavy chain and muscle fiber types, as well as myofiber hypertrophy, appear to be more dependent on intensity than exercise duration. On the other hand, the duration is more prevalent than the exercise intensity in the degree of muscular oxidative adaptation and capillarization. However, significant trends observed in the majority of muscle adaptations to training suggest that the total workload completed during training is the main determinant of the type and extent of peripheral response [6]. Therefore, significant changes occur in the skeletal muscles of sport horses undergoing a training program in order to adapt to the increasing muscular metabolic demand. The organism seeks to offer the animal mechanisms to achieve maximum performance, requiring less energy expenditure and avoiding premature fatigue. Studies on this topic are extremely important since equestrian sports are becoming more common and important all over the world.

\section{References}

1. GUY PS, Snow DH (1997) The effect of training and detraining on muscle composition in the horse. J. Physiol 269(1): 33-51.

2. Leisson K, Jaakma Ü, Seene T (2008) Adaptation of equine locomotor muscle fiber types to endurance and intensive high training speed training. Journal of Equine Veterinary Science 28(7): 395-401.

3. Lindner A, Erginsoy SD, Kissen beck S, Mosen H, Hetzel U, et al. (2013) Effect of different blood-guided conditioning programmes on skeletal muscles ultra e structure and histochemistry of sport horses. Journal of Animal Physiology and Animal Nutrition 97(2): 374-386.

4. Rivero JLL, Ruz A, Korff SM, Estepa JC, Tejero EA, et al. (2007) Effects of intensity and duration of exercise on muscular responses to training of Thoroughbred horses. Journal of Applied Physiology 102(5): 18711882

5. Serrano AL, Rothe EQ, JLL Rivero (2000) Early and long-term changes of equine skeletal muscle in response to endurance training and detraining. Pflügers Arch Eur J Physiol 441(2-3): 263-274. 
6. Tyler CM, Golland LC, Evans DL, Hodgson DR, Rose RJ (1998) Skeletal muscle adaptations to prolonged training, overtraining and detraining in horses. Pflügers Arch Eur J Physiol 436(3): 391-397.

ISSN: 2574-1241

DOI: 10.26717/BJSTR.2019.18.003087

Felipe Gomes Ferreira Padilhaa. Biomed J Sci \& Tech Res

(c) (P) This work is licensed under Creative

Submission Link: https://biomedres.us/submit-manuscript.php

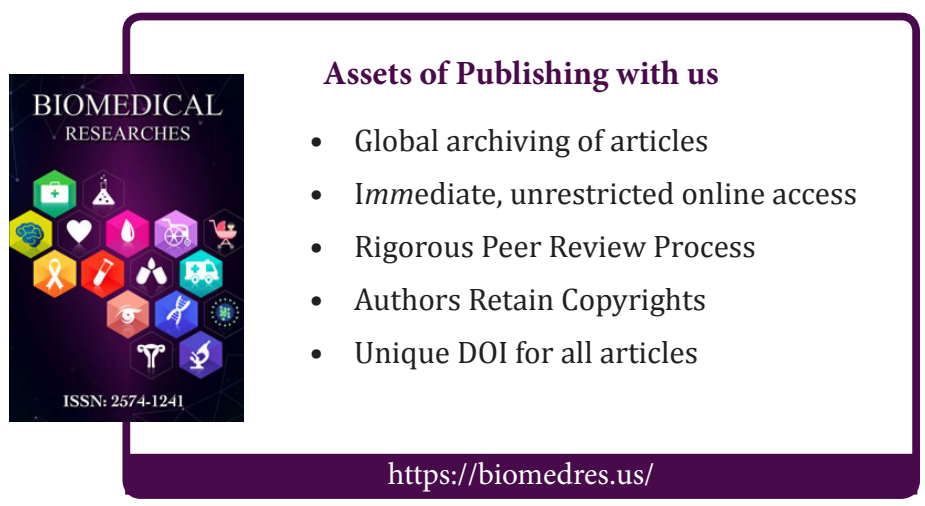

